\title{
Multiple Attribute Comprehensive Evaluation Method Based on Interval Number Aggregation Operators
}

\author{
Zhang Bing-Jiang \\ School of Applied Science, Beijing Information Science and Technology University, Beijing, China \\ Email address: \\ zbj2013ch@163.com \\ To cite this article: \\ Zhang Bing-Jiang. Multiple Attribute Comprehensive Evaluation Method Based on Interval Number Aggregation Operators. American \\ Journal of Applied Mathematics. Vol. 6, No. 2, 2018, pp. 42-47. doi: 10.11648/j.ajam.20180602.13
}

Received: January 31, 2018; Accepted: February 12, 2018; Published: April 27, 2018

\begin{abstract}
The concept of position weight is put forward based on the varied position of different attribute value in the overall distribution of attribute value with the same attribute in multiple attribute and comprehensive assessment issues. What's more, the calculation method of position weight is given and the interval numbers ordered weighted averaging (INOWA) is defined. A comprehensive evaluation method based on position weight of attribute value is put forward. Finally, case study shows that the method is feasible and effective.
\end{abstract}

Keywords: Multiple Attribute Decision Making (MADM), Aggregation Operators, Falling Shadows Method, Position Weight

\section{Introduction}

In the decision-making procedure of multiple attribute issues, the decision maker usually determines the order of superiority and inferiority of different decision-making plans under fuzzy environment. To handle such situation, data instruments such as interval number, triangular fuzzy number and binary correlation coefficient are used to deal with the actual fuzziness. There are lots of fuzzy decision-making methods, such as $\alpha$ cut set method [1-3]. As for the method in literature [1, 2], all values of real numbers have been assigned to $\alpha$, which brings about great inconvenience in application in reality. In literature [3], it is hard to understand the function expression of fuzzy maximum set and fuzzy minimum set. Meanwhile, technicians find it hard to apply to reality. Though the expression of membership function has been adopted for some domestic researches, decision-making information has been made accurate before modeling, which causes the loss of information. Literature [4-7] was based on the theory of correlation coefficient of set pair analysis, putting forward multiple attribute decision-making method from decision-maker weight, principal weight and principal value and binary correlation coefficient. As for these approaches, further study is required in sequencing rules, algorithms and information collection. What's more, principal weight among principals and the weight position among principal values under the same principal are worth great attention. Therefore, the article defines the arithmetic aggregation operators in the interval number information based on algorithm in the internal number and applies operators to multiple attribute and comprehensive evaluation methods. During the process, the calculation method and usage procedures are discussed about position weight among principal values with the same principal.

Aggregation operator is an interesting research topic and a rapidly developing mathematical domain. Yager [8] introduced the ordered weighted averaging (OWA) operator which is a useful tool for aggregating the exact inputs. The key of the OWA operator is to determine its associated weights. O'Hagan [9] was the first to determine OWA operator weights and suggested a maximum entropy method, which formulated the OWA operator weight problem as a constrained nonlinear optimization model with a predefined degree of orness as its constraint and the entropy as its objective function. For a minimum variance method to obtain the minimal variability OWA operator weights, Fuller and Majlender [10] also suggested their idea. Majlender [11] extended the maximum entropy method to Renyi entropy and proposed a maximal Renyi entropy method that produced maximal Renyi entropy OWA weights for a given level of orness. Wang and Parkan [12] introduced a minmax disparity approach which determined the OWA operator weights by 
minimizing the maximum difference between two adjacent weights under a given level of orness. Wang and Luo [13] introduced two new methods for determining the OWA operator weights: the least squares method and the chi-square method. $\mathrm{Xu}$ and $\mathrm{Da}$ [14] established a linear objective-programming procedure for obtaining the OWA weights from observational data under partial weight information. With respect to the hybrid aggregation (HA) operator, they are also used to deal with the problem of a kind of synthesis of all individual. This article defines interval numbers ordered weighted averaging (INOWA) in the light of the operators mentioned earlier. Besides utilize these tools to deal with MADM problem presented fuzziness in form of interval number.

\section{Normal Distribution Interval Numbers and Comparison Between Intervals}

For the multiple attribute fuzzy decision problem, its attribute value can be represented by the interval number. For such intervals, we usually think of a normal distribution.

Definition 1 ([15]) Supposed interval number $\alpha=[a, b]$, if attribute value $r \in[a, b]$ and $r \sim N\left(\mu, \sigma^{2}\right)$, then $[a, b]$ is called normal distribution interval number, written $\beta=\{\mu, \sigma\}$, where according to a normal distribution $3 \sigma$ principle, the desired value and the variance are as follows:

$$
\begin{gathered}
\mu=(a+b) / 2 \\
\sigma=(b-a) / 6
\end{gathered}
$$

Definition 2 ([15]) Supposed arbitrary two normal distribution interval numbers $\beta_{1}=\left\{\mu_{1}, \sigma_{1}\right\}, \beta_{2}=\left\{\mu_{2}, \sigma_{2}\right\}$, then

$$
\begin{aligned}
& \text { If } \mu_{1}<\mu_{2} \text {, then } \beta_{1}<\beta_{2} \text {. } \\
& \text { If } \mu_{1}=\mu_{2}, \text { (1) } \sigma_{1}=\sigma_{2} \text {, then } \beta_{1}=\beta_{2} . \\
& \text { (2) } \sigma_{1}<\sigma_{2} \text {, then } \beta_{1}>\beta_{2} . \\
& \text { (3) } \sigma_{1}>\sigma_{2} \text {, then } \beta_{1}<\beta_{2} .
\end{aligned}
$$

\section{Position Weight and Calculation Method of Position Weight}

In general, the attribute value of each decision-making unit under the same attribution is placed in different position in the overall number distribution. These attribute values in different positions not only reflect the score in a certain attribute, but also display the importance level in all attribute values. To distinguish the importance among these attribute values, we need to calculate the position weight of attribute values belonging to each decision-making unit under the same attribution.

The evaluation result of decision-maker to objective attribute is generally normal distribution. Then we will offer the calculation method of position weight in attribute values that are normally distributed. If attribute values are displayed by interval numbers, the distribution of attribute values in each decision-making unit under the same attribution should be distributed normally. What we do is to find out the center of the normal distribution and compute the distance between the center of attribute values in each decision-making unit and the center of the normal distribution and further get the position of attribute value in each decision-making unit. Calculate the weight of the attribute value according to the location of the attribute value. The closer the location is to the center of the normal distribution, the greater the weight of the attribute value is.

Definition 3 Let $\alpha=[a, b]$ be an interval number, $a \leq b$, then we call

$$
d(a, b)=(a+b) / 2-\beta
$$

as the correlation distance between $\beta$ and interval number $\alpha$.

Definition 4 Let $\alpha_{1 j}=\left[a_{1 j}, b_{1 j}\right], \alpha_{2 j}=\left[a_{2 j}, b_{2 j}\right]$, $\left[a_{m j}, b_{m j}\right], \quad j=1,2, \cdots, n$ are the $m$ attribute values of the same attribute, $d_{1 j}\left(a_{1 j}, b_{1 j}\right), d_{2 j}\left(a_{2 j}, b_{2 j}\right), \cdots, d_{m j}\left(a_{m j}, b_{m j}\right)$, $j=1,2, \cdots, n$ are the distance between the attribute value and the "center" value, and the position weight of the attribute value is calculated by the following expression.

$$
\begin{gathered}
v_{1 j}=d_{1 j}\left(a_{1 j}, b_{1 j}\right) / \sum_{i=1}^{m} d_{i j}\left(a_{i j}, b_{i j}\right), \\
v_{2 j}=d_{2 j}\left(a_{1 j}, b_{1 j}\right) / \sum_{i=1}^{m} d_{i j}\left(a_{i j}, b_{i j}\right), \\
\vdots \\
v_{m j}=d_{m j}\left(a_{m j}, b_{m j}\right) / \sum_{i=1}^{m} d_{i j}\left(a_{i j}, b_{i j}\right),
\end{gathered}
$$

where $j=1,2, \cdots, n$.

\section{Falling Shadows Method and Calculation Method of the Position Weight of Interval Number}

In the comprehensive evaluation of large scale, the interval number with people fuzziness and uncertainty is consistent, which can largely compensate the deficiencies of deterministic values. But the interval number can't be directly applied to the existing comprehensive evaluation methods. However, the set-value statistics theory is an effective way to solve this problem. Fuzzy Sets and falling shadows of Random Sets are introduced in Wang Peizhuang [16]. In this book, we can understand the set-value statistics theory, falling shadows function and the measure to falling shadows. It is applied to handle with fuzzy information and the estimation of 
falling shadows function etc. In general, falling shadows method is by evaluate group decision making problem. However, in this paper, we make a series of evaluative alternative in the same attribute and use position weight to sort alternatives. At the same time, we also use falling shadows function solve the total desired value and deviation in every attribute.

For the judgment matrix $\boldsymbol{A}=\left(\alpha_{i j}\right)_{m \times n}, i=1,2, \cdots, m$, $j=1,2, \cdots, n$, If the $\alpha_{i j}$ is a fuzzy number, then the interval number $\alpha_{i j}=\left[a_{i j}, b_{i j}\right]$ can be used to indicate the comparison result between alternative $i$ and $j$ with respect to every attribute. Then the judgment matrix $\boldsymbol{A}$ is an interval number judgment matrix, where $a_{i j} \leq b_{i j}$. Thus, the different alternatives in the same attribute have formed a sequence, i.e.,

$$
\left\{\left[a_{i 1}, b_{i 1}\right],\left[a_{i 2}, b_{i 2}\right], \cdots,\left[a_{i n}, b_{i n}\right]\right\}, i=1,2, \cdots, m
$$

where $a_{i j}^{\min }=\min \left\{a_{i j}\right\}, \quad b_{i j}^{\max }=\max \left\{b_{i j}\right\}, \quad i=1,2, \cdots, m$, $j=1,2, \cdots, n$, then the comparative result among evaluation alternative $j$ in the same attribute $i$ is a random distribution on $\left[a_{i j}^{\min }, b_{i j}^{\max }\right]$. For arbitrary point $x_{i j}$, the fuzzy coverage scale is defined as

$$
f_{i j}\left(x_{i j}\right)=\frac{1}{n} \sum_{j=1}^{n} f_{x_{i j}}\left(x_{i j}\right)
$$

where

$$
f_{x_{i j}}\left(x_{i j}\right)= \begin{cases}1, & x_{i j} \in\left[a_{i j}, b_{i j}\right] \\ 0, & \text { other }\end{cases}
$$

The desired value of different alternatives in one attribute is

$$
\beta_{i}=\frac{\int_{a_{i j}^{\min }}^{a_{i j}^{\max }} x_{i j} f_{i j}\left(x_{i j}\right) d x}{\int_{a_{i j}^{\min }}^{b_{i j}^{\max }} f_{i j}\left(x_{i j}\right) d x}
$$

According to the algorithms of interval number, by the formula (7) and (8) we can be derived

$$
\int_{a_{i j}^{\min }}^{b_{i j}^{\max }} f_{i j}\left(x_{i j}\right) d x=\frac{1}{n} \sum_{j=1}^{n}\left[b_{i j}-a_{i j}\right]
$$

and

$$
\int_{a_{i j}^{\min }}^{a_{i j}^{\max }} x_{i j} f_{i j}\left(x_{i j}\right) d x=\frac{1}{2 n} \sum_{j=1}^{n}\left[\left(b_{i j}\right)^{2}-\left(a_{i j}\right)^{2}\right]
$$

$$
\beta_{i}=\frac{\sum_{j=1}^{n}\left[\left(b_{i j}\right)^{2}-\left(a_{i j}\right)^{2}\right]}{2 \sum_{j=1}^{n}\left[b_{i j}-a_{i j}\right]}
$$

and its random deviation is

$$
\begin{aligned}
s_{i} & =\int_{a_{i j}^{\min }}^{b_{i j}^{\max }}\left(b_{i j}-a_{i j}\right)^{2} d x \\
& =\frac{1}{3 n} \sum_{j=1}^{n}\left[\left(b_{i j}-a_{i j}\right)^{3}-\left(b_{i j}-a_{i j}\right)^{3}\right]
\end{aligned}
$$

\section{Aggregation Operators}

Consider the normal distribution interval number in one attribute, such as in the $i$ th attribute $i=1,2, \cdots, m$, $\beta_{i j}=\left\{\mu_{i j}, \sigma_{i j}\right\}, \quad j=1,2, \cdots, n$, and sort $\beta_{i j}=\left\{\mu_{i j}, \sigma_{i j}\right\}$, $j=1,2, \cdots, n$ to $\beta_{i j}^{\prime}=\left\{\mu_{i j}^{\prime}, \sigma_{i j}^{\prime}\right\}, j=1,2, \cdots, n$ in accordance with normal distribution. Assume $\beta_{i j}^{\prime \prime}=v_{\left\{\mu_{(j)}, \sigma_{(j)}\right\}} \otimes \beta_{i j}^{\prime}$ $=\left\{v_{\left\{\mu_{(j)}, \sigma_{(j)}\right\}} \mu_{i j}^{\prime}, v_{\left\{\mu_{(j)}, \sigma_{(j)}\right\}} \sigma_{i j}^{\prime}\right\}, j=1,2, \cdots, n$, here when it comes to the same position, adjust the original position weight. It is to say that the original position weight $v=\left(v_{1}, v_{2}, \cdots, v_{n}\right)^{\mathrm{T}}$ has changed as the adjusted position weight $v_{\{\mu, \sigma\}}=\left(v_{\left\{\mu_{(1)}, \sigma_{(1)}\right\}}, v_{\left\{\mu_{(2)}, \sigma_{(2)}\right\}}, \cdots, v_{\left\{\mu_{(n)}, \sigma_{(n)}\right\}}\right)^{\mathrm{T}} \quad$ in $\quad$ a certain form. And $\left\{\mu_{(1)}, \sigma_{(1)}\right\},\left\{\mu_{(2)}, \sigma_{(2)}\right\}, \cdots,\left\{\mu_{(m)}, \sigma_{(m)}\right\}$ is one replacement of $\{1,2, \cdots, m\}$.

Definition 5 The interval numbers ordered weighted averaging (INOWA) is a mapping: $\Omega^{n} \rightarrow \Omega$, such that

$$
\begin{aligned}
\operatorname{INOWA}_{w}\left(\beta_{1 j}^{\prime \prime},\right. & \left.\beta_{2 j}^{\prime \prime}, \cdots, \beta_{m j}^{\prime \prime}\right) \\
& =w_{1} \beta_{1 j}^{\prime \prime} \oplus w_{2} \beta_{2 j}^{\prime \prime} \oplus \cdots \oplus w_{m} \beta_{m j}^{\prime \prime}
\end{aligned}
$$

where $w=\left(w_{1}, w_{2}, \cdots, w_{m}\right)^{\mathrm{T}}$ is the attribute weight vector with $w_{i} \in[0,1], \quad i=1,2, \cdots, m, \quad \sum_{i=1}^{m} w_{i}=1, \quad$ then $\operatorname{INOWA}_{w}\left(\beta_{1 j}^{\prime \prime}, \beta_{2 j}^{\prime \prime}, \cdots, \beta_{m j}^{\prime \prime}\right)$ is call the interval number ordered weighted averaging (INOWA) operator.

From formula (13), we know the result of above-mentioned aggregation operator is still a interval number and the aggregation result is

Thus, the formula (8) can be written as 


$$
\begin{aligned}
& \operatorname{INOWA}_{w}\left(\beta_{1 j}^{\prime \prime}, \beta_{2 j}^{\prime \prime}, \cdots, \beta_{m j}^{\prime \prime}\right) \\
& =\left\{\sum_{i=1}^{m} w_{i} \mu_{i j}^{\prime \prime}, \sqrt{\sum_{i=1}^{m} w_{i}^{2} \sigma_{i j}^{\prime \prime 2}}\right\}(j=1,2, \cdots, n)
\end{aligned}
$$

Finally, assume $\operatorname{INOWA}_{w}\left(\beta_{1 j}^{\prime \prime}, \beta_{2 j}^{\prime \prime}, \cdots, \beta_{m j}^{\prime \prime}\right)=\left\{\mu^{\prime \prime \prime}, \sigma^{\prime \prime \prime}\right\}$ and according to Definition 2, compare them and take an order in MADM problems.

\section{Algorithms}

Based on description of aggregation operators, we put forward to algorithm steps about solve the MADM problems.

Step 1 Based on the falling shadows method, compute the overall desired value and deviation value.

Step 2 According to a practical issue, we should analyze different attribute of the issue. For every attribute, calculate the middle value and respective deviation value and compare the difference between the middle value and desired value. If occur one situation that two or more differences value are equal, we need reallocate position weight in form of the ratio of respective deviation.

Step 3 Calculate the position weight and adjust the proportion of the same weight part.

Step 4 Under every attribute, compute the desired value, i.e. the desired value is that multiply by middle value of interval numbers and respective adjust position weight.

Step 5 Based on the INOWA operator, make use of the result of Step 4 to calculate every alternative value. If alternative values are very close to each other, then we will need compare deviation both them.

Step 6 According to the result of Step 5 and the rule of Definition 2 take an order with every alternative.

\section{Numerical Experiments}

The consumption of furniture is quite common in people's daily life. As for traditional consumption, great importance has been attached to material needs. However, modern consumption ideas include material need and spiritual need, referring to functional need and psychological need, which are actual the objectivity and subjectivity of consumption needs. Objectivity refers to the objective functions in terms of physics, chemistry, physiology and art in consumption, whose variation is small based on different consumers. Subjectivity refers to the influences exerted on by the level of interests, hobbies and psychologies in consumers, which are largely dependent on the world view, social status and literacy of consumers and are quite varied.

As for the selection of indoor furniture set, we attach great importance to functions, outlook design and service. Function includes application, size, material, diversity and comfort. Outlook design includes style tone, stability and modern sense. Service includes the completeness of manuals, after-sale service and past credit.

To select a set of satisfactory furniture, customers will make decisions based on the above three factors and price. It is easy to compare price. However, it is hard to compare the three factors. In this way, it is suggested to apply the evaluation of multiple attribute and comprehensive method to the five furniture sets.

Set the evaluation benchmark as hundred-mark system. The comprehensive scores of five furniture sets $\left\{A_{1}, A_{2}, A_{3}, A_{4}, A_{5}\right\}$ in terms of functions $I_{1}$, outlook design $I_{2}$ and manufacturer's service $I_{3}$ are displayed in Table 1 .

Table 1. Decision-making Matrix.

\begin{tabular}{llll}
\hline \multirow{2}{*}{ Alternatives } & \multicolumn{3}{c}{ Attribute } \\
\cline { 2 - 4 } & $\mathbf{I}_{\mathbf{1}}$ & $\mathbf{I}_{\mathbf{2}}$ & $\mathbf{I}_{\mathbf{3}}$ \\
\hline $\mathrm{A}_{1}$ & {$[84,86]$} & {$[85,87]$} & {$[86,88]$} \\
$\mathrm{A}_{2}$ & {$[85,90]$} & {$[78,82]$} & {$[84,90]$} \\
$\mathrm{A}_{3}$ & {$[82,89]$} & {$[82,84]$} & {$[82,90]$} \\
$\mathrm{A}_{4}$ & {$[78,80]$} & {$[88,90]$} & {$[80,90]$} \\
$\mathrm{A}_{5}$ & {$[85,97]$} & {$[70,80]$} & {$[90,96]$} \\
\hline
\end{tabular}

The expected value and deviation value are calculated according to formula (1) and formula (2) and the results are shown in Table 2.

Table 2. Expected value and Deviation value.

\begin{tabular}{lllllll}
\hline \multirow{2}{*}{ Alternatives } & \multicolumn{7}{c}{ Attribute } \\
\cline { 2 - 7 } & $\mathbf{I}_{\mathbf{1}}$ & \multicolumn{7}{c}{$\mathbf{I}_{\mathbf{2}}$} & \multicolumn{1}{c}{$\mathbf{I}_{\mathbf{3}}$} \\
\cline { 2 - 7 } & $\mu$ & $\sigma$ & $\mu$ & $\sigma$ & $\mu$ & $\sigma$ \\
\hline $\mathrm{A}_{1}$ & 85 & 1 & 86 & 1 & 87 & 1 \\
$\mathrm{~A}_{2}$ & 87.5 & 2.5 & 80 & 2 & 87 & 3 \\
$\mathrm{~A}_{3}$ & 85.5 & 3.5 & 83 & 1 & 86 & 4 \\
$\mathrm{~A}_{4}$ & 79 & 1 & 89 & 1 & 5 & 5 \\
$\mathrm{~A}_{5}$ & 91 & 6 & 75 & 5 & 93 & 3 \\
\hline
\end{tabular}

The central value of the attribute value calculated by formula (11) are 94.46, 88.11 and 93.07 respectively. By formula (3) we get the distance between the attribute value and the central value. As shown in Table 3. For the formula (4), we obtain the position weights of each attribute value. As shown in Table 4.

Table 3. The distance between the attribute value and the central value.

\begin{tabular}{llll}
\hline \multirow{2}{*}{ Alternatives } & \multicolumn{3}{c}{ Attribute } \\
\cline { 2 - 4 } & $\mathbf{I}_{\mathbf{1}}$ & $\mathbf{I}_{\mathbf{2}}$ & $\mathbf{I}_{\mathbf{3}}$ \\
\hline $\mathrm{A}_{1}$ & 9.46 & 2.11 & 6.07 \\
$\mathrm{~A}_{2}$ & 6.96 & 8.11 & 6.07 \\
$\mathrm{~A}_{3}$ & 8.96 & 5.11 & 7.07 \\
$\mathrm{~A}_{4}$ & 15.46 & 0.89 & 8.07 \\
$\mathrm{~A}_{5}$ & 3.46 & 13.11 & 0.07 \\
\hline
\end{tabular}

Table 4. The position weights.

\begin{tabular}{llll}
\hline \multirow{2}{*}{ Alternatives } & \multicolumn{3}{c}{ Attribute } \\
\cline { 2 - 4 } & $\mathbf{I}_{\mathbf{1}}$ & $\mathbf{I}_{\mathbf{2}}$ & $\mathbf{I}_{\mathbf{3}}$ \\
\hline $\mathrm{A}_{1}$ & 0.21 & 0.07 & 0.22 \\
$\mathrm{~A}_{2}$ & 0.16 & 0.28 & 0.22 \\
$\mathrm{~A}_{3}$ & 0.20 & 0.17 & 0.26 \\
$\mathrm{~A}_{4}$ & 0.35 & 0.03 & 0.29 \\
$\mathrm{~A}_{5}$ & 0.08 & 0.45 & 0.01 \\
\hline
\end{tabular}

Next, the attribute values are revised according to the position weights given in Table 4 , and the results are shown in 
Table 5. If the weights of three attributes are set to $0.5,0.3$ and 0.2 respectively, the revision values of the attribute values of each decision unit are shown in Table 6. From the INOWA operator calculation, i.e. formula (14), we get the comprehensive evaluation results. As shown in Table 7.

Table 5. Expected value and deviation value via the position weight.

\begin{tabular}{lllllll}
\hline \multirow{2}{*}{ Alternatives } & \multicolumn{7}{c}{ Attribute } \\
\cline { 2 - 7 } & $\mathbf{I}_{\mathbf{1}}$ & \multicolumn{5}{c}{$\mathbf{I}_{\mathbf{2}}$} \\
\cline { 2 - 7 } & $\mu$ & $\sigma$ & $\mu$ & $\sigma$ & $\mu$ & $\sigma$ \\
\hline $\mathrm{A}_{1}$ & 18.15 & 0.21 & 6.19 & 0.07 & 19.31 & 0.22 \\
$\mathrm{~A}_{2}$ & 13.75 & 0.39 & 22.12 & 0.55 & 19.31 & 0.67 \\
$\mathrm{~A}_{3}$ & 17.29 & 0.71 & 14.46 & 0.17 & 22.23 & 1.03 \\
$\mathrm{~A}_{4}$ & 27.57 & 0.35 & 2.70 & 0.03 & 25.09 & 1.48 \\
$\mathrm{~A}_{5}$ & 7.12 & 0.47 & 33.52 & 2.23 & 0.27 & 0.01 \\
\hline
\end{tabular}

Table 6. Expected value and deviation value via the attribute weight.

\begin{tabular}{lllllll}
\hline \multirow{3}{*}{ Alternatives } & \multicolumn{7}{c}{ Attribute } \\
\cline { 2 - 7 } & $\mathbf{I}_{\mathbf{1}}$ & \multicolumn{5}{c}{$\mathbf{I}_{\mathbf{2}}$} \\
\cline { 2 - 7 } & $\mu$ & $\sigma$ & $\mu$ & $\sigma$ & $\mu$ & $\sigma$ \\
\hline $\mathrm{A}_{1}$ & 9.08 & 0.11 & 1.86 & 0.02 & 3.86 & 0.04 \\
$\mathrm{~A}_{2}$ & 6.87 & 0.20 & 6.64 & 0.17 & 3.86 & 0.13 \\
$\mathrm{~A}_{3}$ & 8.65 & 0.35 & 4.34 & 0.05 & 4.44 & 0.21 \\
$\mathrm{~A}_{4}$ & 13.78 & 0.17 & 0.81 & 0.01 & 5.02 & 0.30 \\
$\mathrm{~A}_{5}$ & 3.55 & 0.23 & 10.06 & 0.44 & 0.05 & 0.00 \\
\hline
\end{tabular}

Table 7. The comprehensive evaluation results.

\begin{tabular}{ll}
\hline Alternative & $\left\{\mu_{A_{i}}, \sigma_{A_{i}}\right\}$ \\
\hline $\mathrm{A}_{1}$ & $\{14.79,0.17\}$ \\
$\mathrm{A}_{2}$ & $\{17.37,0.49\}$ \\
$\mathrm{A}_{3}$ & $\{17.43,0.61\}$ \\
$\mathrm{A}_{4}$ & $\{19.61,0.47\}$ \\
$\mathrm{A}_{5}$ & $\{13.66,0.68\}$ \\
\hline
\end{tabular}

According to the results of Table 8 and Definition 2, we can get the comprehensive ranking result of five furniture sets, namely $A_{4} \succ A_{3} \succ A_{2} \succ A_{1} \succ A_{5}$.

As calculated by Table 2 , the expected value of $A_{3}$ is larger than $A_{2}$, but the deviation value of $A_{3}$ is larger than that of $A_{2}$. In this regard, decision makers need to reconsider the ordering of decision units $A_{2}$ and $A_{3}$.

\section{Conclusion}

In this paper, with respect to multiple attribute decision making problems in which both the attribute weight and position weights take the form of interval numbers, the approach of determining position weight is obtained by one rule that evaluate object things always follow normal distribution. One is comparing the difference between desired values of the same attribute which is obtained by the falling shadows method. The approach is to make evaluation alternatives as one series in an attribute. The other one is obtaining alternative values by analyzing several class of aggregation operator. On the basis of the position weight of the attribute value of the positive distribution, the interval number assembly operator is proposed, and the selection of the five furniture sets is analyzed by using this operator.

\section{Acknowledgements}

The research was supported by the National Natural Science Foundation of China (Grant No. 60972115).

\section{References}

[1] Dubois D, Prade H, "Comment on tolerance analysis using fuzzy set $\mathrm{s}$ and a procedure for multiple aspect decision making”, Int J of System Science. 1978, vol. 9, pp. 357-360.

[2] Dubois D, Prade H, "A review of fuzzy set aggregation connectives", Information Science. 1985, vol. 36, pp. 85-121.

[3] Bonissone P P, "A pattern recognition approach to the problem of linguistic approximation in system analysis", Proc of the IEEE Int Conf on Cybernetics and Society. New York. 1979, pp. 793-798.

[4] Wang X F, "On method of multi-attribute group decision-making under pure linguistic information based on connection number", Control and Decision. 2006, vol. 23, pp. 1180-584.

[5] Liu X M, Zhao K Q, "multiple attributes decision making of intervals based on analysis of the uncertainty of connection number", Fuzzy Systems \& Mathematic. 2010, vol. 24, pp. 141-148.

[6] Wang X F, Wang J Q and Yang E E, "multiple criteria group decision making method based on binary connection number aggregation operators", Fuzzy Systems \& Mathematic. 2013, vol. 28, pp. 1630-1636.

[7] WANG Xin-fan, Wang Jian-qiang and YANG Wu-e, "Multiple criteria group decision making method based on binary connection number aggregation operators", Control and Decision. 2013, vol. 28, pp. 1630-1636.

[8] Yager, R. R, “On OWA aggregation operators in multicriteria decision making", IEEE Transactions on Systems, Man and Cybernetics. 1988, vol. 18, pp. 183-190.

[9] O'Hagan, M, "Aggregating template or rule antecedents in real-time expert systems with fuzzy set logic", in: Proceedings 22nd Annual IEEE Asilomar Conference on Signals, Systems and Computers, Pacific Grove. 1988, pp. 681-689.

[10] Fuller, R. and Majlender, P, "On obtaining minimal variability OWA operator weights”, Fuzzy Sets and Systems. 2003, vol. 136, pp. 203-215.

[11] Majlender, P, "OWA operators with maximal Renyi entropy", Fuzzy Sets and Systems. 2005, vol. 155, pp. 340-360.

[12] Wang, Y. M. and Parkan, C, "A minimax disparity approach for obtaining OWA operator weights", Information Sciences. 2005, vol. 175 , pp. $20-29$.

[13] Wang, Y. M. and Luo, Y, "Two new models for determining OWA operator weights", Computers and Industrial Engineering. 2007, vol. 52, pp. 203-209.

[14] Xu Zeshui and Da Q. L, "The uncertain OWA operator", Int J Fuzzy Intell Syst. 2002, vol. 17, pp. 569-575.

[15] Wang Xinfan and Xiao mansheng, "Approach of group decision based on normal distribution interval number with incomplete information", Control and Decision. 2010, vol. 25, pp. 1494-1506. 
[16] Wang Pei zhuang, "Fuzzy Sets and falling shadows of Random Sets”, Beijing Normal University Press, Beijing, 1985.

[17] LIU Xiu-mei , ZHAO Ke-qin, “On Studying Hybrid Multi-attribute Decision-making with Interval Number and Linguistic Value", Fuzzy Systems and Mathematics. 2014, vol. 28, pp. 113-118.

[18] Slah Benyoussef, "Clustering Problem with Fuzzy Data: Empirical Study for Financial Distress Firms", American Journal of Applied Mathematics. 2015, vol. 3, pp. 75-80.

[19] Cai-Li Zhou, "A New Fuzzy-Valued Additive Measure", American Journal of Applied Mathematics. 2015, vol. 3, pp. 259-264.

[20] Fang Liu, Wei-Guo Zhang and Yu-Fan Shang, "A group decision-making model with interval multiplicative reciprocal matrices based on the geometric consistency index",
Computers \& Industrial Engineering. 2016, vol. 101, pp. 184-193.

[21] Pankaj Gupta, Mukesh Kumar Mehlawat and Nishtha Grover, "Intuitionistic fuzzy multi-attribute group decision-making with an application to plant location selection based on a new extended VIKOR method", Information Sciences. 2016, 370 371, pp. 184-203.

[22] Depeng Fan, Shouhai Wang, "Application of Fuzzy Data Fusion in Storage Environment Security Monitoring", American Journal of Applied Mathematics. 2016, vol. 4, pp. 197-203.

[23] ZHANG Mei-jin, WANG Ying-ming, CHEN Sheng-qun and LI Kai, "Approach for multiple attribute decision making under interval uncertainty considering preference reversal", Control and Decision. 2016, vol. 31, pp. 2019-2024. 